\title{
TENSORING GENERALIZED CHARACTERS WITH THE STEINBERG CHARACTER
}

\author{
G. HISS AND A. ZALESSKI
}

\begin{abstract}
Let $\mathbf{G}$ be a reductive connected algebraic group over an algebraic closure of a finite field of characteristic $p$. Let $F$ be a Frobenius endomorphism on $\mathbf{G}$ and write $G:=\mathbf{G}^{F}$ for the corresponding finite group of Lie type.

We consider projective characters of $G$ in characteristic $p$ of the form $S t \cdot \beta$, where $\beta$ is an irreducible Brauer character and $S t$ the Steinberg character of $G$.

Let $M$ be a rational G-module affording $\beta$ on restriction to $G$. We say that $M$ is $G$-regular if for every $F$-stable maximal torus $\mathbf{T}$ distinct weight spaces of $M$ are nonisomorphic $\mathbf{T}^{F}$-modules. We show that if $M$ is $G$-regular of dimension $d$, then the lift of $S t \cdot \beta$ decomposes as a sum of $d$ regular characters of $G$.
\end{abstract}

\section{INTRODUCTION}

Let $K$ be an algebraically closed field of prime characteristic $p, \mathbf{C}$ the field of complex numbers and $H$ a finite group. It is well known that every projective $K H$-module $M$ lifts to characteristic zero, that is, there is a $\mathbf{C ~} H$-module $L$ whose character at $p^{\prime}$-elements coincides with the Brauer character of $M$. The multiplicities of the irreducible constituents of $L$ are called the decomposition numbers of $M$. One of the classical problems of the modular representation theory of finite groups is to determine the decomposition numbers, especially when $M$ is indecomposable.

The problem is intensely studied for quasi-simple groups of Lie type with $p$ being the defining characteristic, the latest contribution seems to be in Chastkofsky [5] where one can also find the relevant bibliography. See also Humphreys' book [12, Ch.9] for a survey of this circle of problems.

In this paper we study the decomposition numbers for the products of the Steinberg character with certain generalized Brauer characters. In many instances these are the Brauer characters of projective modules. According to the philosophy developed in Ballard [1], Humphreys [12], Jantzen [13] and Chastkofsky [5], this may highlight the problem of computing the decomposition numbers for principal indecomposable modules.

Let $G$ be a quasi-simple group of Lie type in defining characteristic $p$. Recall there is a unique irreducible $K G$-module of defect 0 and its lift to characteristic 0 is called the Steinberg module. Its character is called the Steinberg character. This can also be defined via the character formula [4, 71.19], and this formula can be used to define the Steinberg character for $G:=\mathbf{G}^{F}$ where $\mathbf{G}$ is an arbitrary reductive connected algebraic group with Frobenius endomorphism $F$. We denote the Steinberg character of $G$ by $S t$ (or $S t_{G}$ ). We

2000 Mathematics Subject Classification. 20C33, 20C20, 20 G05.

Key words and phrases. Projective characters, Chevalley groups, Steinberg character. 
study irreducible constituents of $S t \cdot \beta$ where $\beta$ is a generalized (Brauer) character of $G$. As $S t$ vanishes at the $p$-singular elements of $G$, the values of $\beta$ on such elements are immaterial.

In this paper we are only concerned with decomposing the characters $S t \cdot \beta$ as sum of ordinary irreducible characters of $G$ and do not touch the problem of decomposing them in terms of the Brauer characters of principal indecomposable modules. Of course one can also try to decompose $\psi \cdot S t$ where $\psi$ is an ordinary character. In [9] we consider a special case with $\psi$ the Weil characters of symplectic or unitary groups. In this paper we use some machinery developed in [9].

In order to state our results we introduce some basic notation.

Below $\mathbf{G}$ is a reductive connected algebraic group over $K, F$ a Frobenius endomorphism of $\mathbf{G}, G=\mathbf{G}^{F}=\{x \in \mathbf{G}: F(x)=x\}$, and $\mathbf{T}$ is an $F$-stable maximal torus of $\mathbf{G}, T=\mathbf{T}^{F}$. (It is customary to call $T$ a maximal torus of $G$.) The action of $F$ on $\mathbf{T}$ induces the dual action of $F$ on $\Omega=\operatorname{Hom}\left(\mathbf{T}, K^{\times}\right)$, the set of rational irreducible representations of $\mathbf{T}$, which is an integral lattice. This action extends to a linear transformation of $\bar{\Omega}:=\Omega \otimes \mathbf{C}$. It is known that all eigenvalues of $F$ on $\bar{\Omega}$ have the same absolute value usually denoted by $q$. This $q$ determines $F$ and one often uses the notation $G(q)$ for $\mathbf{G}^{F}$. (Notice that $q$ is a prime power unless $G \in\left\{{ }^{2} B_{2}(q),{ }^{2} G_{2}(q),{ }^{2} F_{4}(q)\right\}$.)

In the Deligne-Lusztig theory of characters of groups of Lie type, the irreducible characters of $G$ partition into sets $\mathcal{E}_{s}$ where $s$ runs over semisimple conjugacy classes of the dual group $G^{*}$. Therefore, every class function $f$ on $G$ can be written as $\sum_{s} f_{s}$ where $f_{s}$ is the projection of $f$ onto the span of the characters in $\mathcal{E}_{s}$. In this paper the central role is played by so called regular characters. These are, by definition, constituents of a Gelfand-Graev character. If $\mathbf{G}$ is with connected center then every $\mathcal{E}_{s}$ contains a unique regular character, which coincides with $\Gamma_{s}$ where $\Gamma$ is the Gelfand-Graev character of $G$. If $Z(\mathbf{G})$ is not connected, the situation is more complicated, however, for all $s$ occurring in our results, $\mathcal{E}_{s}$ contains a unique regular character (see Section 3 ).

The main result of this paper is the following theorem.

Theorem 1.1. Let $\phi$ be a generalized character of $G$. Suppose that the restriction of $\phi$ to $T$ is a multiplicity free proper character of $T$ for every maximal torus $T$ of $G$. Then the character $\phi \cdot$ St is a sum of exactly $\phi(1)$ distinct regular characters.

Our proof of Theorem 1.1 is based on the analysis of $(\phi \cdot S t)_{s}$, and in fact we prove that this projection is a regular character. We do this in a slightly more general form by refining the hypothesis of the theorem in terms of $s$.

Let $M$ be a rational $\mathbf{G}$-module. We say that $M$ is $G$-regular, if for every maximal $F$ stable torus $\mathbf{T}$ distinct weight spaces of $M$ are non-isomorphic $T$-modules. Alternatively, we can fix $\mathbf{T}$ and apply this condition to conjugates of maximal tori of $G$ in $\mathbf{T}$.

Theorem 1.2. Let $M$ be an irreducible $\mathbf{G}$-module, $G=\mathbf{G}^{F}$ and let $\chi$ be the Brauer character of $\left.M\right|_{G}$. Suppose that $M$ is $G$-regular. Then

(1) $\chi \cdot S t_{G}$ is a sum of $d$ regular characters where $d=\operatorname{dim} M$;

(2) the set of the multiplicities of the irreducible constituents in $\left.M\right|_{G} \otimes S t_{G}$ (disregarding repetitions) coincides with the set of the weight multiplicities of $M$. 
Observe that the number of irreducible constituents in item (2) of Theorem 1.2 is greater than the number of weights of $M$, so we need to disregard the repetitions to obtain the coincidence.

Given $\mathbf{G}$ and $M$, there are only finitely many $q$ such that $M$ is not $G(q)$-regular; in $\S 6$, using some results of Guralnick and Tiep [8], we provide an upper bound for such $q$ in terms of the highest weight of $M$ for simple groups G. Seitz [14] showed that the adjoint module is $G(q)$-regular if $q>5$ (he did not introduce any name for this property).

The following result allows one to detect regular character constituents in $\phi \cdot S t$ even if $\phi$ is not $G$-regular.

Theorem 1.3. Let $s \in G^{*}$ be semisimple and let $\phi$ be a generalized character of $G$. Suppose that $\left(\left.\phi\right|_{T}, \theta\right) \in\{0,1\}$ for all $(\mathbf{T}, \theta)$ corresponding to the conjugacy class of $s$. Then $\phi \cdot S t$ contains at most one constituent from $\mathcal{E}_{s}$, and this is a regular character.

Theorem 1.1 is an immediate consequence of Theorem 1.3. Moreover, the latter also implies Theorem 1.2 as soon as one shows that the Brauer character of $M$ is a sum of generalized characters satisfying the assumption of Theorem 1.1. This can be shown by using some generalized characters $s_{\mu}$ attached to every weight $\mu$ of $\mathbf{G}$. These $s_{\mu}$ have been introduced by Wong [18], and we call them here Wong characters (see Section 5 for the definition). If $M$ is $G$-regular then the restriction $\left.s_{\mu}\right|_{T}$ is multiplicity free for every weight $\mu$ of $M$ and for every maximal torus $T$ of $G$. Another advantage of Wong characters is that $s_{\mu} \cdot S t$ is the character of a projective module if $\mu$ is small. Namely, Ballard [1, Corollary 4.5] proves this assuming that $\ln (\mu)<q$ where $\ln$ is a certain 'weight length function'.

The above notion of $G$-regularity is meaningful for any T-module. This allows us to define $G$-regular weights as follows. Let $\mu$ be a weight, $W$ the Weyl group of $\mathbf{G}$ and $W \mu$ the $W$-orbit of $\mu$. Consider 1-dimensional T-modules $U_{\nu}$ for all weights $\nu \in W \mu$. We say that $\mu$ is $G$-regular if for every $\nu \neq \nu^{\prime}$ in $W \mu$ and for every maximal torus $T$ of $G$ the spaces $U_{\nu}$ and $U_{\nu^{\prime}}$ are non-isomorphic $T$-modules. As an immediate consequence of Theorem 1.1 we have:

Theorem 1.4. If $\mu$ is $G$-regular then the character $\left.s_{\mu}\right|_{G} \cdot$ St is a sum of exactly $s_{\mu}(1)=|W \mu|$ distinct regular characters.

We could provide more explicit information on the decomposition numbers in Theorem 1.4. However, this would lead to technicalities which we would prefer to avoid in this paper.

The fact that the $s_{\mu}$ of $G(q)$ are defined in terms of the algebraic group $\mathbf{G}$, and behave in a sense uniformly on $q$, seems to be significant. It is rather surprising that our proof makes no use of the representation theory of algebraic groups, and is somehow based on general ideas.

Naturally, the question arises whether Theorem 1.1 is a proper generalization of Theorem 1.4. Equivalently, is it true that $\phi$ is a sum of some $s_{\mu}$ with non-negative integer coefficients? At the moment we do not have any result in this direction.

\section{Tensoring With the Steinberg character}

We denote by $(\cdot, \cdot)$ the inner product of functions on $G$. We write $W$ or $W(\mathbf{G})$ for the Weyl group of $\mathbf{G}$. We set $W(T):=N_{\mathbf{G}}(\mathbf{T})^{F} / T$ and $W(T)_{\theta}=C_{W(T)}(\theta)$ where $\theta$ is an 
irreducible character of $T$. In addition, $\varepsilon_{\mathbf{G}}:=(-1)^{r}$ where $r$ is the relative rank (or $F_{q^{-}}$ rank) of a connected algebraic group $\mathbf{G}$, which therefore is meaningful for $\mathbf{T}$ (consult $[7$, pp. 64,66]).

For every $F$-stable maximal torus $\mathbf{T}$ and an irreducible character $\theta$ of $T:=\mathbf{T}^{F}$ Deligne and Lustzig introduce a generalized character $R_{\mathbf{T}, \theta}$, see $[7,11.14]$, which plays a part in this paper. If the pairs $(\mathbf{T}, \theta)$ and $\left(\mathbf{T}^{\prime}, \theta^{\prime}\right)$ are $G$-conjugate then $R_{\mathbf{T}, \theta}=R_{\mathbf{T}^{\prime}, \theta^{\prime}}$ and $\left(R_{\mathbf{T}, \theta}, R_{\mathbf{T}, \theta}\right)=$ $\left|W(T)_{\theta}\right|$, otherwise $\left(R_{\mathbf{T}, \theta}, R_{\mathbf{T}^{\prime}, \theta^{\prime}}\right)=0([7, \mathrm{p} .88])$. This is called the orthogonality relations for Deligne-Lustzig characters.

The following lemma is a part of the reasoning in [3, p.242].

Lemma 2.1. Let $\phi$ be any class function on $G$ and St the Steinberg character. Then

$$
\phi \cdot \mathrm{St}=\sum_{(\mathbf{T}, \theta)} \frac{\left(\left.\phi\right|_{T}, \theta\right)}{\left|W(T)_{\theta}\right|} \varepsilon_{\mathbf{G}} \varepsilon_{\mathbf{T}} R_{T, \theta}
$$

where the summation ranges over representatives of $G$-conjugacy classes of pairs $(\mathbf{T}, \theta)$ in $G$.

Lemma 2.2. Let $\phi, \psi$ be class functions of $G$. Then

$$
(\phi \cdot S t, \psi \cdot S t)_{G}=\sum_{(\mathbf{T}, \theta)} \frac{\left(\left.\phi\right|_{T}, \theta\right)_{T} \cdot\left(\left.\psi\right|_{T}, \theta\right)_{T}}{\left|W(T)_{\theta}\right|},
$$

where the summation ranges over representatives of $G$-conjugacy classes of pairs $(\mathbf{T}, \theta)$ in $G$. In particular, if $(\phi, \psi)_{T}=0$ for all maximal tori $T$ in $G$ then $(\phi \cdot S t, \psi \cdot S t)_{G}=0$.

Proof. Observe that $(\phi, \psi)_{T}=0$ implies $(\theta, \psi)_{T}=0$ for every irreducible constituent $\theta$ of $\phi$. Furthermore,

$$
(\phi \cdot S t, \psi \cdot S t)=\sum_{(\mathbf{T}, \theta)} \frac{\left(\left.\phi\right|_{T}, \theta\right)}{\left|W(T)_{\theta}\right|}\left(\varepsilon_{G} \varepsilon_{\mathbf{T}} R_{\mathbf{T}, \theta}, S t \cdot \psi\right)_{G}=\sum_{(\mathbf{T}, \theta)} \frac{\left(\left.\phi\right|_{T}, \theta\right)_{T}}{\left|W(T)_{\theta}\right|}\left(\theta^{G}, \psi\right)_{G}
$$

which implies the formula in the statement since $\left(\varepsilon_{\mathbf{G}} \varepsilon_{\mathbf{T}} R_{\mathbf{T}, \theta}, S t \cdot \psi\right)_{G}=\left(\varepsilon_{\mathbf{G}} \varepsilon_{\mathbf{T}} R_{\mathbf{T}, \theta}\right.$. $S t, \psi)_{G}=\left(\theta^{G}, \psi\right)_{G}$ (see [3, Proposition 7.4.5]) and $\left(\theta^{G}, \psi\right)_{G}=\left(\theta,\left.\psi\right|_{T}\right)_{T}$.

One of the fundamental notions of the character theory of Chevalley groups is that of a geometric conjugacy of pairs $(\mathbf{T}, \theta)$ where $\mathbf{T}$ is an $F$-stable maximal torus and $\theta$ is an irreducible character of $T=\mathbf{T}^{F}$, see for instance [3,7]. There is a bijective correspondence between geometric conjugacy classes of pairs $(\mathbf{T}, \theta)$ and $F^{*}$-stable semisimple conjugacy classes in the dual group $\mathbf{G}^{*}([7,13.13])$. This is based on the notion of duality of simple algebraic groups as explained in $[7,13.10]$. If $\mathbf{G}^{*}$ is dual to $\mathbf{G}$ then there is a Frobenius morphism $F^{*}$ of $\mathbf{G}^{*}$ such that $G=\mathbf{G}^{F}$ and $G^{*}:=\mathbf{G}^{* F^{*}}$ are of the same order. (Below we omit the star from $F^{*}$ as we do not expect any confusion from this.) Duality includes a bijection $T \rightarrow T^{*}$ between $G$-conjugacy classes of maximal tori $T$ in $G$ and $G^{*}$-conjugacy classes of maximal tori $T^{*}$ in $G^{*}$ such that $W(T) \cong W\left(T^{*}\right)$ (and also $W(\mathbf{G}) \cong W\left(\mathbf{G}^{*}\right)$ ). If $C$ is an $F$-stable semisimple conjugacy class, then $C \cap G^{*}$ is non-empty, so $C$ is determined by $C \cap G^{*}$ which is a set of elements of $G^{*}$ that are $\mathbf{G}^{*}$-conjugate but not always $G^{*}$-conjugate. In addition, $C \cap G^{*}$ is a union of conjugacy classes in $G^{*}$. Therefore, a geometric conjugacy 
class of pairs $(\mathbf{T}, \theta)$ can be further partitioned into subclasses parametrized by $G^{*}$-conjugacy classes of semisimple elements, see $[7,13.13]$. So given a semisimple element $s \in G^{*}$ we denote by $[s]$ the set of pairs $(\mathbf{T}, \theta)$ corresponding to the $G^{*}$-conjugacy class of $s$. If $C_{\mathbf{G}^{*}}(s)$ is connected then an element $s^{\prime} \in G^{*}$ is $G^{*}$-conjugate to $s$ if and only if it is $\mathbf{G}^{*}$-conjugate to $s$. Therefore, if $C_{\mathbf{G}^{*}}(s)$ is connected then $[s]$ is a geometric conjugacy class of pairs $(\mathbf{T}, \theta)$. In addition, we denote by $[s]_{G}$ some set of representatives of the $G$-conjugacy classes in $[s]$.

Lemma 2.3. Let $s \in G^{*}$ be a semisimple element, $\mathbf{S}=C_{\mathbf{G}^{*}}(s)$ and $S=\mathbf{S}^{F}$. In addition, let $\mathbf{S}^{0}$ be the connected component of $\mathbf{S}$ and $S^{0}=S \cap \mathbf{S}^{0}$. Then the cardinality of $[s]_{G}$ is equal to the number of $S$-conjugacy classes of $F$-stable maximal tori in $\mathbf{S}^{0}$, and does not exceed the number of F-conjugacy classes in $W_{s}^{0}$, the Weyl group of $\mathbf{S}^{0}$.

Proof. There is a bijection between $G$-conjugacy classes of pairs $(\mathbf{T}, \theta)$ and $G^{*}$-conjugacy classes of pairs $\left(s, \mathbf{T}^{*}\right)$ with $s \in T^{*}$, see $[7,13.13]$. If $s$ is fixed then saying that $(\mathbf{T}, \theta)$ belongs to $[s]$ means that $\mathbf{T}^{*}$ contains this fixed $s$, and $(\mathbf{T}, \theta) \in[s]$ is $G$-conjugate to $\left(\mathbf{T}^{\prime}, \theta^{\prime}\right)$ is equivalent to saying that $\left(s, \mathbf{T}^{*}\right)$ is $G^{*}$-conjugate to $\left(s, \mathbf{T}^{*}\right)$. Therefore, the cardinality of $[s]_{G}$ is equal to the number of $S$-conjugacy classes of $F$-stable maximal tori in $\mathbf{S}$. As $\mathbf{T}^{*}$ is connected and hence is contained in $\mathbf{S}^{0}$, the first assertion of the lemma follows. The number of $S^{0}$-conjugacy classes of $F$-stable maximal tori in $\mathbf{S}^{0}$ is equal to the number of $F$-conjugacy classes in $W_{s}^{0}$, see $[7,3.23]$. As $S^{0} \subset S$, the lemma follows.

Lemma 2.4. Let $H \subset \mathbf{G}$ be reductive connected algebraic groups and $F$ a Frobenius endomorphism of $\mathbf{G}$ such that $F(H)=H$. Let $g \in N_{\mathbf{G}^{F}}(H)$ be a semisimple element. Then $g$ stabilizes an F-stable maximal torus of $H$.

Proof. This is proved in $[16,5.16]$ for $H$ being semisimple. So let $T^{\prime}$ be an $F$-stable maximal torus of $H^{\prime}$, the semisimple part of $H$. Then $H=H^{\prime} \cdot Z(H)^{0}$, and $T:=T^{\prime} \cdot Z(H)^{0}$ is a maximal torus in $H$ ([3, Section 1.8]). Obviously, $Z(H)$ is $F$-stable and $g$-stable, and hence so is $T$.

Let $s, s^{\prime} \in G^{*}$ be semisimple elements, $(\mathbf{T}, \theta) \in[s]$ and $\left(\mathbf{T}^{\prime}, \theta^{\prime}\right) \in\left[s^{\prime}\right]$. If $s, s^{\prime}$ are not conjugate in $G^{*}[7,14.41]$ then $R_{\mathbf{T}, \theta}$ and $R_{\mathbf{T}^{\prime}, \theta^{\prime}}$ have no common irreducible constituents. The set of irreducible characters not orthogonal to every $R_{\mathbf{T}, \theta} \in[s]$ is denoted by $\mathcal{E}_{s}$. An important fact of the Deligne-Lusztig theory is that every irreducible character of $G$ belongs to $\mathcal{E}_{s}$ for some semisimple element $s \in G^{*}$ and $\mathcal{E}_{s} \cap \mathcal{E}_{s^{\prime}}=\emptyset$ if $s, s^{\prime}$ are not conjugate in $G^{*}$. In other words, $\operatorname{Irr} G=\cup \mathcal{E}_{s}$ is a partition, where $s$ runs over representatives of semisimple conjugacy classes in $G^{*}$. It follows that, for every semisimple $G^{*}$-conjugacy class in $G^{*}$, expression (1) uniquely determines the partial sums

$$
\pi_{s}(\phi)=\sum_{(\mathbf{T}, \theta) \in[s]_{G}} \frac{\left(\left.\phi\right|_{T}, \theta\right)}{\left|W(T)_{\theta}\right|} \varepsilon_{\mathbf{G}} \varepsilon_{\mathbf{T}} R_{T, \theta} .
$$

(Here $\pi_{s}(\phi)$ depends on the $G^{*}$-conjugacy class of $s$ rather than on $s$. Note that we used the notation $(\phi \cdot S t)_{s}$ in place of $\pi_{s}(\phi)$ in the introduction.) Therefore $\phi \cdot S t=\sum \pi_{s}(\phi)$ with the summation over representatives of the semisimple conjugacy classes in $G^{*}$. Observe that $\left(\pi_{s}(\phi), \pi_{s^{\prime}}(\phi)\right)=0$ if $s, s^{\prime}$ are not conjugate in $G^{*}$. 
Lemma 2.5.

$$
\left(\pi_{s}(\phi), \pi_{s}(\phi)\right)=\sum_{(\mathbf{T}, \theta) \in[s]_{G}} \frac{\left(\left.\phi\right|_{T}, \theta\right)_{T}^{2}}{\left|W(T)_{\theta}\right|} .
$$

Proof. Follows from Lemma 2.2.

Lemma 2.6. $\pi_{s}(\phi) \neq 0$ if and only if there exists a pair $(\mathbf{T}, \theta) \in[s]$ such that $\theta$ is a constituent of $\left.\phi\right|_{T}$.

Proof. As distinct functions $R_{\mathbf{T}, \theta}$ are linear independent [7, 11.15], $\pi_{s}(\phi) \neq 0$ if and only if the coefficient of some $R_{\mathbf{T}, \theta}$ in (2) is non-zero.

Lemma 2.7. If $\phi$ is a generalized character of $G$, then so are $\phi \cdot$ St and $\pi_{s}(\phi)$.

Proof. The first claim is trivial. Recall that $\operatorname{Irr} G=\cup \mathcal{E}_{s}$ is a partition. It follows from the definition of $\mathcal{E}_{s}$ that $R_{\mathbf{T}, \theta}$ for $(\mathbf{T}, \theta) \in[s]$ is a linear combination of characters in $\mathcal{E}_{s}$. Therefore, if $\phi \cdot S t=\sum_{i} m_{i} \chi_{i}\left(m_{i} \in \mathbf{Z}, \chi_{i} \in \operatorname{Irr} G\right)$, then $\pi_{s}(\phi)=\sum_{i} m_{i} \chi_{i}$ where $\chi_{i} \in \mathcal{E}_{s}$. So we conclude that $\pi_{s}(\phi)$ is a generalized character.

Lemma 2.8. Let $\mathbf{G}$ be a reductive connected group with Frobenius endomorphism $F$. Then $\sum \frac{1}{|W(T)|}=1$ where the sum is over $G$-conjugacy classes of $F$-stable maximal tori in $\mathbf{G}$.

Proof. This is a particular case of [3, Proposition 7.8.6] (see the remark after Lemma 4.1 below for details).

\section{3. $G$-Regular generalized CharaCters}

We keep the notation of the previous section. In particular, for a semisimple element $s \in G^{*}$ we set $\mathbf{S}=C_{\mathbf{G}^{*}}(s)$ and $S=\mathbf{S}^{F}$. In addition, $\mathbf{S}^{0}$ is the connected component of $\mathbf{S}$ and $S^{0}=S \cap \mathbf{S}^{0}$. Following the notation in [7, 2.4 and 14.40], we set $W_{s}\left(T^{*}\right):=N_{\mathbf{S}}^{F}\left(\mathbf{T}^{*}\right) / T^{*}$ and $W_{s}^{0}\left(T^{*}\right):=N_{\mathbf{S}^{0}}^{F}\left(\mathbf{T}^{*}\right) / T^{*}$. In this section, a generalized character $\phi$ is called s-regular if $0 \leq\left(\left.\phi\right|_{T}, \theta\right) \leq 1$ for every $(\mathbf{T}, \theta) \in[s]$.

Lemma 3.1. Let $\phi$ be a generalized character. Suppose that $\phi$ is s-regular. Then $\pi_{s}(\phi)$ is an irreducible character or 0 . In the former case $\left(\left.\phi\right|_{T}, \theta\right)=1$ for all $(\mathbf{T}, \theta) \in[s], S=S^{0}$ and

$$
\pi_{s}(\phi)=\sum_{(\mathbf{T}, \theta) \in[s]_{G}} \frac{1}{\left|W(T)_{\theta}\right|} \varepsilon_{\mathbf{G}} \varepsilon_{\mathbf{T}} R_{\mathbf{T}, \theta}=\sum_{\mathbf{T}^{*} \subset \mathbf{S}} \frac{1}{\left|W_{s}^{0}\left(T^{*}\right)\right|} \varepsilon_{\mathbf{G}} \varepsilon_{\mathbf{T}} R_{\mathbf{T}, \theta},
$$

where the second sum runs over $S^{0}$-conjugacy classes of $F$-stable maximal tori in $\mathbf{S}^{0}$. In addition, $W_{s}^{0}\left(T^{*}\right)=W_{s}\left(T^{*}\right)$, the cardinality of $[s]_{G}$ is equal to the number of $S^{0}$-conjugacy classes of F-stable maximal tori in $\mathbf{S}^{0}$, and hence to the number of $F$-conjugacy classes in $W_{s}^{0}$.

Proof. Suppose that $\pi_{s}(\phi) \neq 0$. By the definition of an $s$-regular character, $0 \leq\left(\left.\phi\right|_{T}, \theta\right) \leq$ 1 , so from (2) and Lemma 2.5 we have

$$
\left(\pi_{s}(\phi), \pi_{s}(\phi)\right) \leq \sum_{(\mathbf{T}, \theta) \in[s]_{G}} \frac{1}{\left|W(T)_{\theta}\right|}=\sum_{(\mathbf{T}, \theta) \in[s]_{G}} \frac{1}{\left|W_{s}\left(T^{*}\right)\right|}
$$


where $\mathbf{T}^{*} \subset \mathbf{S}$ is dual to $\mathbf{T}$. The equality holds as $W(T)_{\theta}=W_{s}\left(T^{*}\right)$, see [3, page 289]. By Lemma 2.3 , the cardinality of $[s]_{G}$ does not exceed the number of $F$-stable $S^{0}$-conjugacy classes of maximal tori in $\mathbf{S}$. As $W_{s}^{0}\left(T^{*}\right) \subseteq W_{s}\left(T^{*}\right)$, hence $\left|W_{s}\left(T^{*}\right)\right| \geq\left|W_{s}^{0}\left(T^{*}\right)\right|$, we get

$$
\left(\pi_{s}(\phi), \pi_{s}(\phi)\right) \leq \sum_{\mathbf{T}^{*} \subset \mathbf{S}^{0}} \frac{1}{\left|W_{s}\left(T^{*}\right)\right|} \leq \sum_{\mathbf{T}^{*} \subset \mathbf{S}^{0}} \frac{1}{\left|W_{s}^{0}\left(T^{*}\right)\right|}=1 .
$$

The former sum is over representatives of $F$-stable $S$-conjugacy classes of maximal tori in $\mathbf{S}^{0}$ and the latter sum is over representatives of $F$-stable $S^{0}$-conjugacy classes of maximal tori in $\mathbf{S}$. The equality in (4) follows from Lemma 2.8 applied to $\mathbf{S}^{0}$. As $\pi_{s}(\phi)$ is a non-zero generalized character (Lemma 2.7), this inequality implies $\left(\pi_{s}(\phi), \pi_{s}(\phi)\right)=1$. In addition, $\pi_{s}(\phi)(1)>0$ as $\varepsilon_{\mathbf{G}} \varepsilon_{\mathbf{T}} R_{\mathbf{T}, \theta}(1)=\theta^{G}(1) / S t(1)>0$, see [3]. So the first assertion of the lemma follows. Furthermore, the equality $\left(\pi_{s}(\phi), \pi_{s}(\phi)\right)=1$ implies that all inequalities in the proof are in fact equalities. Therefore, $\left(\left.\phi\right|_{T}, \theta\right)=1$ for all $(\mathbf{T}, \theta) \in[s]_{G},\left|W_{s}\left(T^{*}\right)\right|=\left|W_{s}^{0}\left(T^{*}\right)\right|$ (hence $W_{s}\left(T^{*}\right)=W_{s}^{0}\left(T^{*}\right)$ ), and that the number of terms in the right hand side of (3) is the same as in the last sum in (4). It follows that $\pi_{s}(\phi)=\sum_{(\mathbf{T}, \theta) \in[s]_{G}} \frac{1}{\mid W(T)_{\theta}} \varepsilon_{\mathbf{G}} \varepsilon_{\mathbf{T}} R_{\mathbf{T}, \theta}$. By Lemma 2.4, the equalities $W_{s}\left(T^{*}\right)=W_{s}^{0}\left(T^{*}\right)$ for all $F$-stable maximal tori $\mathbf{T}^{*}$ in $\mathbf{S}$ implies that $S=S^{0}$.

Remark. The assertion that $S=S^{0}$ is not equivalent to $\mathbf{S}=\mathbf{S}^{0}$. For instance, consider a torus $T$ of order 3 in $S L(3,2)$.

We recall the correspondence between the $G$-conjugacy classes of $F$-stable maximal tori and $F$-conjugacy classes in $W$, the Weyl group of $\mathbf{G}$, see [7, 3.23]. Fix an $F$-stable maximal torus $\mathbf{T}_{0}$, and identify $W$ with $N:=N_{\mathbf{G}}(\mathbf{T})$. Let $w \in W$, and let $n_{w} \in N$ be an element such that $w=n_{w} \mathbf{T}$. By Lang's theorem, there is $g \in \mathbf{G}$ such that $g^{-1} F(g)=n_{w}$. We wish to fix one of such $g$ for every $w$, so we write $g$ as $g_{w}$. Then $T_{w}:=g_{w} \mathbf{T} g_{w}^{-1}$ is an $F$-stable maximal torus in $\mathbf{G}$. The tori $T_{w}$ and $T_{w^{\prime}}$ are $G$-conjugate if and only if $w, w^{\prime} \in W$ are in the same $F$-conjugacy class, that is, $w^{\prime}=v^{-1} w F(v)$ for some $v \in W$. Moreover, if $w$ runs over representatives of the $F$-conjugacy classes in $W$, tori $T_{w}$ runs over representatives of all $G$-conjugacy classes of $F$-stable maximal tori in $\mathbf{G}$.

In the next lemma this is applied to $\mathbf{S}^{0}=C_{\mathbf{G}^{*}}^{0}(s)$ for some semisimple $s \in G^{*}$.

\section{Lemma 3.2.}

$$
\sum_{\mathbf{T}^{*} \subset \mathbf{S}} \frac{1}{\left|W_{s}^{0}\left(T^{*}\right)\right|} \varepsilon_{\mathbf{G}} \varepsilon_{\mathbf{T}} R_{\mathbf{T}, \theta}=\frac{1}{\left|W_{s}^{0}\right|} \sum_{w \in W_{s}^{0}} \varepsilon_{\mathbf{G}} \varepsilon_{\mathbf{T}_{w}} R_{\mathbf{T}_{w}, \theta} .
$$

Here the pairs $(\mathbf{T}, \theta)$ and $\left(\mathbf{T}^{*}, s\right)$ are dual, and the left hand side sum runs over $S^{0}$-conjugacy classes of F-stable maximal tori in $\mathbf{S}^{0}$. At the right hand side $T_{w}$ is the torus obtained from $w \in W_{s}^{0}$ and the sum runs over the whole $W_{s}^{0}$ (so there are repetitions of equal terms).

Proof. In view of the correspondence described prior the lemma, the left hand side can be written as

$$
\sum_{\mathbf{T}_{w}^{*}} \frac{1}{\left|W_{s}^{0}\left(T_{w}^{*}\right)\right|} \varepsilon_{\mathbf{G}} \varepsilon_{\mathbf{T}_{w}} R_{\mathbf{T}_{w}, \theta}
$$

where the summation runs over representatives of $F$-conjugacy classes in $W_{s}^{0}$. Next we rearrange this by repeating the term $\frac{1}{\left|W_{s}^{0}\left(T_{w}^{*}\right)\right|} \varepsilon_{\mathbf{G}} \varepsilon_{\mathbf{T}_{w}} R_{\mathbf{T}_{w}, \theta}$ exactly $a_{w, F}$ times where $a_{w, F}$ is 
the size of the $F$-conjugacy class in $W_{s}^{0}$ that contains $w$. Then we get

$$
\sum_{w \in W_{s}^{0}} \frac{1}{a_{w, F}\left|W_{s}^{0}\left(T_{w}^{*}\right)\right|} \varepsilon_{\mathbf{G}} \varepsilon_{\mathbf{T}_{w}} R_{\mathbf{T}_{w}, \theta}
$$

with the summation over the whole $W_{s}^{0}$. Note that $a_{w, F}\left|W_{s}^{0}\left(T_{w}^{*}\right)\right|=\left|W_{s}^{0}\right|$, see Carter $[3$, Proposition 3.3.6], so the lemma follows.

An irreducible character $\rho$ of $G$ is called regular if it is a constituent of a Gelfand-Graev representation of $G$. If $\mathbf{G}$ is with connected center then there is a unique Gelfand-Graev representation and every $\mathcal{E}_{s}$ contains exactly one regular character. In general, if $\mathbf{G}$ is an arbitrary reductive connected group, then there are several Gelfand-Graev representations $([7,14.29])$, and $\mathcal{E}_{s}$ may contain several regular characters. However, if $S=S^{0}$ then $\mathcal{E}_{s}$ contain a unique regular character, see the remark after Lemma 3.3.

Lemma 3.3. Suppose that $\phi$ is an s-regular generalized character. Then $\pi_{s}(\phi)$ is either 0 or a regular (irreducible) character of $G$.

Proof. By Lemmas 3.1 and 3.2, we have

(5) $\quad \pi_{s}(\phi)=\sum_{(\mathbf{T}, \theta) \in[s]_{G}} \frac{\varepsilon_{\mathbf{G}} \varepsilon_{\mathbf{T}}}{\left|W(T)_{\theta}\right|} R_{\mathbf{T}, \theta}=\sum_{\mathbf{T}^{*} \subset \mathbf{S}} \frac{\varepsilon_{\mathbf{G}^{\mathbf{S}}} \varepsilon_{\mathbf{T}}}{\left|W_{s}^{0}\left(T^{*}\right)\right|} R_{\mathbf{T}, \theta}=\frac{1}{\left|W_{s}^{0}\right|} \sum_{w \in W_{s}^{0}} \varepsilon_{\mathbf{G}^{-}} \varepsilon_{\mathbf{T}_{w}} R_{\mathbf{T}_{w}, \theta}$

where the second sum ranges over $S^{0}$-conjugacy classes of $F$-stable maximal tori $\mathbf{T}^{*}$ in $\mathbf{S}$.

The right hand side expression coincides with the function introduced by Digne and Michel [7, 14.40]. By [7, 14.48,14.49], this function (in general) is a proper character $\chi_{s}$ and $\left(\chi_{s}, \chi_{s}\right)=\left|\left(W_{s} / W_{s}^{0}\right)^{F}\right|$, see $[7,14.43]$, and the irreducible constituents of $\chi_{s}$ are constituents of the sum of the Gelfand-Graev characters. In our situation, $\pi_{s}(\phi)=\chi_{s}$ is irreducible (Lemma 3.1), and hence $\pi_{s}(\phi)$ is a regular character.

Remark. By Lemma 3.1, if $\phi$ is $G$-regular then $S=S^{0}$. It follows that $\mathcal{E}_{s}$ contain a unique regular character $\rho_{s}$ which can be defined by the formula $\sum_{(\mathbf{T}, \theta) \in[s]_{G}} \frac{\varepsilon_{\mathbf{G}} \varepsilon_{\mathbf{T}}}{\left|W(T)_{\theta}\right|} R_{\mathbf{T}, \theta}$. Indeed, by $[7,14.46]$, every irreducible constituent of any Gelfand-Graev character occurs as a constituent of $\chi_{s}$ from the proof of Lemma 3.3. As $S=S^{0}$, the character $\chi_{s}$ is irreducible $([7,14.41])$. So $\rho_{s}=\chi_{s}$ is unique. (However, $\rho_{s}$ is a constituent of every Gelfand-Graev character $([7,14.44])$.

Lemma 3.4. Suppose that $\phi$ is an s-regular generalized character such that $\pi_{s}(\phi) \neq 0$. Then $\pi_{s}(\phi)(1)=\left|G^{*}: S\right|_{p^{\prime}} \cdot|S|_{p}$ where $|S|_{p}$ is the p-part of $|S|$ and $\left|G^{*}: S\right|_{p^{\prime}}=\mid G^{*}$ : $S|/| G^{*}:\left.S\right|_{p}$.

Proof. By Lemma 3.3, $\pi_{s}(\phi)=\frac{1}{\left|W_{s}^{0}\right|} \sum_{w \in W_{s}^{0}} \varepsilon_{\mathbf{G}} \varepsilon_{\mathbf{T}_{w}} R_{\mathbf{T}_{w}, \theta}$ and the lemma follows by the same reasoning as in in the proof of [3, Theorem 8.4.9]. (In fact, the only place of the proof that uses the assumption made in [3] that the center of $\mathbf{G}$ is connected is the equality $\sum_{(\mathbf{T}, \theta) \in[s]_{G}} \frac{1}{\left|W(T)_{\theta}\right|} \varepsilon_{\mathbf{G}} \varepsilon_{\mathbf{T}} R_{\mathbf{T}, \theta}=\sum_{\mathbf{T}^{*} \subset \mathbf{S}} \frac{1}{\left|W_{s}^{0}\left(T^{*}\right)\right|} \varepsilon_{\mathbf{T}} R_{\mathbf{T}, \theta}$, which holds in our situation by Lemma 3.1.)

Theorem 3.5. If $\phi$ is an s-regular generalized character of $G$ then $\pi_{s}(\phi)$ is either 0 or a regular character in $\mathcal{E}_{s}$, and $\pi_{s}(\phi)(1)=\left|G^{*}: S\right|_{p^{\prime}} \cdot|S|_{p}$. In addition, if $\pi_{s}(\phi) \neq 0$ then $C_{\mathbf{G}^{0}}^{F}(s)=C_{\mathbf{G}}^{F}(s)$. 
Proof. This follows from Lemmas 3.3 and 3.4, the additional claim is proved in Lemma 3.1 .

Remark. The additional claim of Theorem 3.5 is useful as it shows that for certain $s$ no generalized character $\phi$ can be $s$-regular.

In the following result we do not assume that $\phi$ is $s$-regular.

Theorem 3.6. Suppose that $\mathbf{S}$ is connected (or $S=S^{0}$ ) and $\left(\left.\phi\right|_{T}, \theta\right)=k$ for all $(\mathbf{T}, \theta) \in[s]$. Then $\pi_{s}(\phi)=k \cdot \rho_{s}$ where $\rho_{s}$ is the regular character in $\mathcal{E}_{s}$.

Proof. It follows from (2) that $\pi_{s}(\phi)=k \cdot \sum_{(\mathbf{T}, \theta) \in[s]_{G}} \frac{1}{\left|W(T)_{\theta}\right|} \varepsilon_{\mathbf{G}} \varepsilon_{\mathbf{T}} R_{\mathbf{T}, \theta}$. If $\mathbf{S}$ is connected (or, by Lemma 2.4, if $S=S^{0}$ ), then $W_{s}^{0}(T)=W_{s}(T)$, and by Lemma 2.3, the number of terms in this sum is equal to the number of $S^{0}$-conjugacy classes of $F$-stable maximal tori in $\mathbf{S}^{0}$. Hence

$$
\sum_{(\mathbf{T}, \theta) \in[s]_{G}} \frac{1}{\left|W(T)_{\theta}\right|} \varepsilon_{\mathbf{G}^{-}} \varepsilon_{\mathbf{T}} R_{\mathbf{T}, \theta}=\sum_{\mathbf{T}^{*} \subset \mathbf{S}^{0}} \frac{1}{\left|W_{s}^{0}\left(T^{*}\right)_{\theta}\right|} \varepsilon_{\mathbf{G}} \varepsilon_{\mathbf{T}} R_{\mathbf{T}, \theta} .
$$

By Lemma 3.2, the right hand side coincides with the function in $[7,14.40]$. The reasoning at the end of the proof of Lemma 3.3 shows that this is a regular character of $G$.

Theorem 3.7. If $\phi$ is a G-regular generalized character then $\phi \cdot$ St is a summand of the Gelfand-Graev character. In addition, the set of pairs $(\mathbf{T}, \theta)$ such that $\left(\left.\phi\right|_{T}, \theta\right) \neq 0$ is a union of classes $[s]$.

Proof. We have that $\phi \cdot S t=\sum \pi_{s}(\phi)$, where the summation ranges over representatives of the semisimple conjugacy classes in $G^{*}$. As $\phi$ is $G$-regular, $\left.\phi\right|_{T}$ is multiplicity free for every maximal torus $T$, so $\left(\left.\phi\right|_{T}, \theta\right) \leq 1$ for every pair $(\mathbf{T}, \theta)$. By Lemma $2.3, \pi_{s}(\phi)$ for every $s$ is either 0 or a regular character. As $\left(\pi_{s}(\phi), \pi_{s^{\prime}}(\phi)\right)=0$ if $s, s^{\prime}$ are not conjugate in $G^{*}$, it follows that $\left.\phi\right|_{T} \cdot S t$ is multiplicity free, which implies the first part of the theorem. In addition, if $(\mathbf{T}, \theta) \in[s]$ then, by Lemma $2.3,\left(\left.\phi\right|_{T}, \theta\right) \neq 0$ implies that $\left(\left.\phi\right|_{T^{\prime}}, \theta^{\prime}\right)=1$ for all $\left(\mathbf{T}^{\prime}, \theta^{\prime}\right) \in[s]$, again by Lemma 2.3 .

Theorem 1.4 is a particular case of Theorem 3.7.

\section{Decomposition of $\psi \cdot S t$ For $G$-Regular Character $\psi$}

Let $\psi$ be any class function of the semisimple classes of $G$. We denote by $\psi^{e}$ its extension to $G$ by setting $\psi^{e}(s u)=\psi(s)$ whenever $u s=s u$ and $u$ is unipotent. As every $g \in G$ is uniquely expressed as $g=s u$ for $s$ semisimple, $u$ unipotent and $s u=u s$, this is a well defined function on $G$. Observe that $\psi^{e}$ is a generalized character if and only if $\psi$ is a generalized Brauer character $[4,18.12]$ (this follows from Brauer's induction theorem). If $\psi$ is a Brauer character then $\psi^{e}$ is called the Brauer lift of $\psi$.

In this section we use the Curtis duality (or the Curtis-Alvis duality) on the vector space of class function on $G$. In particular, the duality is an isometry, that is, a linear map that preserves the inner product of class functions, see [3, Theorem 8.2.1]. 
Lemma 4.1. $\psi^{e}$ and $\psi^{e} \cdot$ St are Curtis dual. In addition,

$$
\left(\psi^{e}, \psi^{e}\right)=\left(\psi^{e} \cdot S t, \psi^{e} \cdot S t\right)=\sum \frac{\left(\left.\psi\right|_{T},\left.\psi\right|_{T}\right)}{|W(T)|} .
$$

Proof. By Carter [3, Proposition 7.6.4] and (2) above, we have:

$$
\psi^{e}=\sum_{(\mathbf{T}, \theta)} \frac{(\psi, \theta)_{T}}{W(T)_{\theta}} R_{\mathbf{T}, \theta} \quad \text { and } \quad \psi^{e} \cdot S t=\sum_{(\mathbf{T}, \theta)} \frac{(\psi, \theta)_{T}}{W(T)_{\theta}} \varepsilon_{\mathbf{G}} \varepsilon_{\mathbf{T}} R_{\mathbf{T}, \theta}
$$

where the summation ranges over all $G$-orbits of pairs $(\mathbf{T}, \theta)$. Deligne and Lusztig [6, p.540] shows that the Curtis dual of $R_{\mathbf{T}, \theta}$ is $\varepsilon_{\mathbf{G}_{\mathbf{T}}} \varepsilon_{\mathbf{T}} R_{\mathbf{T}, \theta}$ (and conversely). As the Curtis duality is an isometry, the first equality of the lemma follows. The second one is [3, Proposition 7.6.8].

Remark. If $\psi=1_{G}$, the principal character, then $\psi=\psi^{e}$ and $\psi^{e} \cdot S t=S t$. So $1=\left(\psi^{e}, \psi^{e}\right)=\sum \frac{1}{|W(T)|}$, the formula stated in Lemma 2.8 .

Lemma 4.2. If $\psi$ is a G-regular generalized character then $(\psi \cdot S t, \psi \cdot S t)=\psi(1)$.

Proof. By Lemma 4.1, $\left(\psi^{e}, \psi^{e}\right)=\sum \frac{\left(\left.\psi\right|_{T},\left.\psi\right|_{T}\right)}{|W(T)|}$. As $\psi$ is $G$-regular, $\left.\psi\right|_{T}$ is multiplicity free for any $T$, so we have that $\left(\left.\psi\right|_{T},\left.\psi\right|_{T}\right)=\psi(1)$. As $\sum \frac{1}{|W(T)|}=1$, we have $\left(\psi^{e}, \psi^{e}\right)=\psi(1)$. By Lemma 4.1, $\left(\psi^{e}, \psi^{e}\right)=(\psi \cdot S t, \psi \cdot S t)$, so the lemma follows.

Corollary 4.3. If $\psi$ is a $G$-regular generalized character then $\psi \cdot$ St is the sum of exactly $\psi(1)$ regular characters.

Recall that an irreducible character is called semisimple if it is the Curtis dual of $\rho$ or $-\rho$ where $\rho$ is a regular character.

Corollary 4.4. If $\psi$ is a $G$-regular generalized character then $\psi^{e}$ is a linear combination of exactly $\psi(1)$ distinct semisimple characters with coefficients 1 or -1 .

Proof. By Lemma 4.3, we can write $\psi \cdot S t=\sum_{i=1}^{\psi(1)} \rho_{s_{i}}$ where $s_{i}$ are suitable semisimple elements from distinct conjugacy classes in $G^{*}$. The Curtis dual of $\psi \cdot S t$ is $\psi^{e}$ (Lemma 4.1) and the Curtis dual of $\rho_{s}$ is $\chi_{s}$ or $-\chi_{s}$ where $\chi_{s}$ is a semisimple character. So the result follows as $\rho_{s}$ is a unique regular character in $\mathcal{E}_{s}$, see the remark after Lemma 3.3.

Example. Let $G=G L(n, q)$ where $q>2$, and let $\psi$ be the Brauer character of the natural $G L(n, q)$-module. Then $\left.\psi\right|_{T}$ is multiplicity free for every maximal torus $T$ of $G$, therefore, $\psi$ is $G$-regular. By Corollaries 4.2 and $4.4, \psi \cdot S t$ and $\psi^{e}$ are multiplicity free and $\left(\psi^{e}, \psi^{e}\right)=n$. These facts are known from Lusztig's book [10] (see also [15, Corollary 1.4]). To some extent, our results have an origin in Lusztig's work [10] of 1974, although the machinery of the Deligne-Lusztig theory we use here has been developed later.

Proof of Theorem 1.1 We have that $\phi \cdot S t=\sum_{s} \pi_{s}(\phi)$ where $s$ runs over $G^{*}$-conjugacy classes of semisimple elements in $G^{*}$. It follows from the assumption that $\phi$ is $s$-regular for every $s$. By Theorem 3.5, each $\pi_{s}(\phi)$ is a regular character. As the functions $\pi_{s}$ are orthogonal to each other, the result follows. 


\section{WONG CHARACTERS}

The generalized characters $s_{\mu}$ were introduced by Wong [18], and we call them here Wong characters. (However, Wong considers only non-twisted groups and one has to consult [12] for the general case.)

Let $\mathbf{G}=\mathbf{G}(K)$ be a reductive connected algebraic group over $K$ where $K$ is the algebraic closure of a finite field. Let $\mathbf{T}$ be an $F$-stable maximal torus in $\mathbf{G}$, and $W=N_{\mathbf{G}}(\mathbf{T}) / \mathbf{T}$ the Weyl group of $\mathbf{G}$. Weights of $\mathbf{G}$ are defined as rational homomorphisms $\mathbf{T} \rightarrow K^{\times}$. They form a group, in fact, a $\mathbf{Z}$-lattice, for which it is customary to use the additive notation. We say that a weight $\mu$ of $\mathbf{G}$ is dominant if the restriction of $\mu$ to $\mathbf{G}^{\prime}$ is a dominant weight of $\mathbf{G}^{\prime}$, the maximal semisimple subgroup of $\mathbf{G}$. Let $\mu$ be a weight of $\mathbf{G}$ and $W_{\mu}$ the stabilizer of $\mu$ in $W$. Generalized characters $s_{\mu}$ can be defined as follows. Consider an irreducible G-module $M$ with highest weight $\mu$ and, for a weight $\nu$ let $M_{\nu}=\{x \in M: t x=\nu(t) x\}$ be the $\nu$-weight space in $M$. Consider $S_{\mu}:=\sum_{\nu \in O} M_{\nu}$ where $O=W / W_{\mu}$ denotes the $W$-orbit of $\mu$, so the number of summands is $\left|W: W_{\mu}\right|$. As $\operatorname{dim} M_{\mu}=1$, it follows that $\operatorname{dim} S_{\mu}=\left|W: W_{\mu}\right|$. Let $\mathbf{C}$ denote the complex number field. As $K^{\times}$is a quasi-cyclic group, there is an injective homomorphism $h: K^{\times} \rightarrow \mathbf{C}$, which we fix. This defines for us the Brauer character $\beta_{\mu}$ of $\mathbf{T}$ on $S_{\mu}$ (as $\mathbf{T}$ is the union of finite subgroups). Note that $\beta_{\mu}$ is stable under $N_{\mathbf{G}}(\mathbf{T})$. As every semisimple element $s \in \mathbf{G}$ is conjugate to an element $t \in \mathbf{T}$, and any two conjugates of $s$ in $\mathbf{T}$ are conjugate in $N_{\mathbf{G}}(\mathbf{T})$, one obtains a well defined function $s_{\mu}$ on the semisimple conjugacy classes of $\mathbf{G}$. Its restriction to $G$ is the Wong character of $G$ attached to $\mu$. We do not expect any confusion from keeping the same notation $s_{\mu}$ for functions on $\mathbf{G}$ and on $G$.

The following lemma is obvious:

Lemma 5.1. Let $M$ be a $\mathbf{G - m o d u l e ~ a n d ~} \beta$ its Brauer character. Then $\beta=\sum_{\mu} m_{\mu} s_{\mu}$ where $\mu$ runs over the dominant weights of $M$ and $m_{\mu}$ is the multiplicity of the weight $\mu$ in $M$.

Lemma 5.2. (Wong) Every Wong character is an integer linear combination of irreducible Brauer characters of $\mathbf{G}$.

Proof. This follows by induction. Recall that $\nu<\mu$ for weights $\mu, \nu$ means that $\nu=$ $\mu-\sum a_{i} \alpha_{i}$ where $\alpha_{i}$ are simple roots and $a_{i}$ are non-negative integers. Let $\mu$ be a dominant weight. There are finitely many dominant weights $\nu$ such that $\nu<\mu$. So we can assume that the lemma is true for all dominant weights $\nu$ such that $\nu<\mu$. Consider an irreducible module $M$ of highest weight $\mu$, and let $\beta_{\mu}$ be its Brauer character. If $\mu$ is the only dominant weight in $M$ then all weights of $M$ are in $W \mu$ so $s_{\mu}=\beta_{\mu}$. Otherwise, $s_{\mu}=\beta_{\mu}-\sum m_{\nu} s_{\nu}$ where the summation runs over the dominant weights $\nu$ of $M$ distinct from $\mu$ and $m_{\nu}$ is the multiplicity of $\nu$ in $M$. It is well known that $\nu<\mu$. So the lemma follows by induction.

Proof of Theorem 1.2 Let $\beta$ be the Brauer character of $M$. Then $\beta=\sum m_{\nu} s_{\nu}$ where the summation ranges over the dominant weights of $M$ and $m_{\nu}$ is the multiplicity of $\nu$. Hence $\beta \cdot S t=\sum m_{\nu} s_{\nu} \cdot S t$. As $M$ is $G$-regular, $\left(\left.s_{\mu}\right|_{T},\left.s_{\nu}\right|_{T}\right)_{T}=0$ for all maximal tori $T$ in $G$ and all weights $\mu \neq \nu$ of $M$. By Lemma $2.2,\left(s_{\mu} \cdot S t, s_{\nu} \cdot S t\right)_{G}=0$. Furthermore, if $M$ is $G$-regular then every weight $\nu$ of $M$ is $G$-regular. By Theorem 3.7, every $s_{\nu} \cdot S t$ is the sum of regular characters, each occurring with multiplicity 1 . Therefore, a regular character that is a constituent of $s_{\nu} \cdot S t$ occurs in $\beta \cdot S t$ with multiplicity $m_{\nu}$. Moreover, the 
number of the irreducible constituents of $s_{\nu} \cdot S t$ is equal to $s_{\nu}(1)$. This coincides with the number of weights in the orbit $W \nu$. As $\operatorname{dim} M=\beta(1)=\sum_{\nu} m_{\nu} \cdot s_{\nu}(1)$, the result follows.

Remark. In general, $s_{\mu} \cdot S t$ is not necessarily a character of $G$. Indeed, consider $G=$ $S L(2, q)$ for $q>5$ and $\mu=(q+1) \omega$ where $\omega$ is the fundamental weight for $\mathbf{G}$. Let $T_{1}, T_{2}$ be maximal tori of $G$ of orders $q-1, q+1$, respectively. Then $\left.s_{\mu}\right|_{T_{2}}=2 \cdot 1_{T_{2}}$ and $\left.s_{\mu}\right|_{T_{1}}=\tau^{2}+\tau^{-2}$ for some faithful character $\tau$ of $T_{1}$. Recall that $\left(R_{\mathbf{T}, \theta}, 1_{G}\right)=1$ if $\theta=1_{T}$ and equals zero otherwise [3, 7.4.1]. Therefore, it follows from Lemma 2.1 that $\left(s_{\mu} \cdot S t, 1_{G}\right)=\varepsilon_{\mathbf{G}_{\mathbf{G}}} \varepsilon_{\mathbf{T}_{2}}=-1$. So $s_{\mu} \cdot S t$ is not a proper character. This example also shows that $\phi \cdot S t$ is not necessarily a character of $G$ if $\phi$ is a generalized character such that $\left.\phi\right|_{T}$ is a proper character of $T$ for every maximal torus $T$ of $G$.

Remark. Humphreys [11] proves the following formula for $s_{\mu} \cdot S t$ :

$$
s_{\mu} \cdot S t=\frac{1}{\left|W_{\mu}\right|} \sum_{w \in W} \varepsilon_{\mathbf{G}} \varepsilon_{\mathbf{T}_{w}} R_{\mathbf{T}_{w}, \theta_{w}} .
$$

(See [11] for notation.) However, this nice formula is not in use in this paper as formula (1) better fits our purpose.

\section{EfFective Bound}

In this section $\mathbf{G}$ is a simple algebraic group. Here we mimic Guralnick and Tiep [8, $5.1,5.2]$. Let $\Omega$ be the weight lattice for $\mathbf{G}$ and $(\cdot, \cdot)$ the standard bilinear form on $\Omega$, see [2]. For $\omega \in \Omega$ we set $\|\omega\|=\sqrt{(\omega, \omega)}$. Define $l=\min \Omega$ to be the minimum value $\|\omega\|$ for $\omega \in \Omega$.

Lemma 6.1. Let $G$ be one of the groups ${ }^{2} B_{2}(q),{ }^{2} F_{4}(q),{ }^{2} G_{2}(q)$, and let $0 \neq \omega \in \Omega$. If $q>8,9,16$ and $t=3,5,6$, respectively, then $\|F(\omega)\|>\frac{q}{t}\|\omega\|$.

Proof. We proceed case-by-case. Let $p$ be the defining characteristic for $G$, so $p=3$ if $G={ }^{2} G_{2}(q)$ and $p=2$ otherwise. Recall that $q=p^{s} \sqrt{p}$ for some integer $s \geq 0$. Let $\alpha, \beta$ be simple roots of $\mathbf{G}$ with $|\alpha|<|\beta|$ and let $y=a \alpha+b \beta$. Then $F(y)=\frac{q}{\sqrt{p}} b \alpha+q \sqrt{p} a \beta=$ $\frac{q}{\sqrt{p}}(b \alpha+p a \beta)$, see $[17, \S 10]$. Let $\varepsilon_{1}, \ldots, \varepsilon_{r}$ be the basis in the weight space introduced in $[2$, Planchees].

Consider the case ${ }^{2} B_{2}$. Then $\alpha_{1}=\varepsilon_{1}-\varepsilon_{2}$ and $\alpha_{2}=\varepsilon_{2}$. Hence $y=a \alpha_{1}+b \alpha_{2}=$ $a \varepsilon_{1}+(b-a) \varepsilon_{2}$ and $(y, y)=2 a^{2}-2 a b+b^{2}$. Similarly, $F(y)=\left(\sqrt{q^{2} / 2}\right)\left(2 b \alpha_{1}+a \alpha_{2}\right)$ so $(F(y), F(y))=\left(q^{2} / 2\right)\left(8 b^{2}-4 a b+a^{2}\right)$. It follows that $(F(y), F(y))>\left(q^{2} / 8\right) \cdot(y, y)$. Indeed, $(F(y), F(y))=\left(q^{2} / 8\right) \cdot\left(32 b^{2}-16 a b+4 a^{2}\right)$ and $32 b^{2}-16 a b+4 a^{2}>2 a^{2}-2 a b+b^{2}$, as this is equivalent to $31 b^{2}-14 a b+2 a^{2}>0$ which is true as the discriminant $14^{2}-4 \cdot 2 \cdot 31=$ $196-248<0$. Therefore, $\|F(y)\|>\frac{q}{\sqrt{8}}\|y\|$.

Consider the case ${ }^{2} G_{2}$. Then $y=a\left(\varepsilon_{1}-\varepsilon_{2}\right)+b\left(-2 \varepsilon_{1}+\varepsilon_{2}+\varepsilon_{3}\right)=(a-2 b) \varepsilon_{1}+(b-a) \varepsilon_{2}+b \varepsilon_{3}$. So $(y, y)=(a-2 b)^{2}+(a-b)^{2}+b^{2}=2 a^{2}-6 a b+6 b^{2}$. Hence $(F(y), F(y))=\left(q^{2} / 3\right)$. $\left(2 b^{2}-18 a b+54 a^{2}\right)$. It follows that $(F(y), F(y))>\left(q^{2} / 33\right) \cdot(y, y)$. Indeed, $(F(y), F(y))=$ $\left(q^{2} / 33\right) \cdot\left(22 b^{2}-198 a b+594 a^{2}\right)$ and $22 b^{2}-198 a b+594 a^{2}>2 a^{2}-6 a b+6 b^{2}$, as this is equivalent to $16 b^{2}-192 a b+592 a^{2}>0$ which is true as the discriminant $192^{2}-4 \cdot 16 \cdot 592=$ $36864-37888<0$. It follows that $\|F(y)\|>\frac{q}{\sqrt{33}}\|y\|$. 
Consider the case ${ }^{2} F_{4}$. Then $\alpha_{1}=\varepsilon_{2}-\varepsilon_{3}$ and $\alpha_{2}=\varepsilon_{3}-\varepsilon_{4}, \alpha_{3}=\varepsilon_{4}$ and $\alpha_{4}=\left(\varepsilon_{1}-\varepsilon_{2}-\right.$ $\left.\varepsilon_{3}-\varepsilon_{4}\right) / 2$. Let $z=a \alpha_{1}+b \alpha_{2}+c \alpha_{3}+d \alpha_{4}=a\left(\varepsilon_{2}-\varepsilon_{3}\right)+b\left(\varepsilon_{3}-\varepsilon_{4}\right)+c \varepsilon_{4}+d\left(\varepsilon_{1}-\varepsilon_{2}-\varepsilon_{3}-\varepsilon_{4}\right) / 2=$ $\frac{d}{2} \varepsilon_{1}+\left(a-\frac{d}{2}\right) \varepsilon_{2}+\left(b-a-\frac{d}{2}\right) \varepsilon_{3}+\left(c-b-\frac{d}{2}\right) \varepsilon_{4}$. Therefore, $(z, z)=\left(\frac{d}{2}\right)^{2}+\left(a-\frac{d}{2}\right)^{2}+\left(b-a-\frac{d}{2}\right)^{2}+(c-$ $\left.b-\frac{d}{2}\right)^{2}=d^{2}+2 a^{2}+2 b^{2}+c^{2}-a d-(b-a) d-(c-b) d-2 b c-2 a b=2 a^{2}+2 b^{2}+c^{2}+d^{2}-c d-2 a b-2 b c$. As $\left|\alpha_{1}\right|=\left|\alpha_{2}\right|>\left|\alpha_{3}\right|=\left|\alpha_{4}\right|$, we have that $F(z)=\frac{q}{\sqrt{2}}\left(2 d \alpha_{1}+2 c \alpha_{2}+b \alpha_{3}+a \alpha_{4}\right)$, and hence $(F(z), F(z))=\frac{q^{2}}{2}\left(8 d^{2}+8 c^{2}+b^{2}+a^{2}-a b-8 c d-4 b c\right):=\frac{q^{2}}{2} x$. We show that if $m>9$ then $m x>(z, z)$. We have

$m x-(z, z)=(m-2)\left(a^{2}+b^{2}\right)+(8 m-1)\left(c^{2}+d^{2}\right)-(8 m-1) c d-(m-2) a b-2(2 m-1) b c$.

Express

$$
\begin{gathered}
-(m-2) a b=\left(a \sqrt{m-2}-\frac{\sqrt{m-2}}{2} b\right)^{2}-(m-2) a^{2}-\frac{m-2}{4} b^{2}, \\
-(8 m-1) c d=\left(\frac{\sqrt{8 m-1}}{2} c-d \sqrt{8 m-1}\right)^{2}-\frac{8 m-1}{4} c^{2}-(8 m-1) d^{2}, \\
-2(2 m-1) b c=\left(b \frac{\sqrt{3(m-2)}}{2}-\frac{2(2 m-1)}{\sqrt{3(m-2)}} c\right)^{2}-\frac{3(m-2)}{4} b^{2}-\frac{4(2 m-1)^{2}}{3(m-2)} c^{2} .
\end{gathered}
$$

Next substitute these expressions into the above formula. Then we have

$$
\begin{aligned}
m x- & (z, z)=c^{2}\left(\frac{3(8 m-1)}{4}-\frac{4(2 m-1)^{2}}{3(m-2)}\right)+\left(a \sqrt{m-2}-\frac{\sqrt{m-2}}{2} b\right)^{2}+ \\
& +\left(\frac{\sqrt{8 m-1}}{2} c-d \sqrt{8 m-1}\right)^{2}+\left(\frac{\sqrt{3(m-2)}}{2} b-\frac{2(2 m-1)}{\sqrt{3(m-2)}} c\right)^{2} .
\end{aligned}
$$

The coefficient of $c^{2}$ is non-negative if and only if $\frac{3(8 m-1)}{4} \geq \frac{4(2 m-1)^{2}}{3(m-2)}$. This inequality is valid for $m \geq 10$. Therefore, $\|F(z)\| \geq \frac{q}{\sqrt{20}}\|z\|$.

Proposition 6.2. Define $k=1 / 3,1 / 5,1 / 6$ for $G={ }^{2} B_{2}(q),{ }^{2} F_{4}(q), G={ }^{2} G_{2}(q)$, respectively, and set $k=1$ otherwise. Let $\mathbf{T}$ be an $F$-stable torus of $\mathbf{G}, T=\mathbf{T}^{F}$ and $\mu \neq 0$ a weight. Then

where $l=\min (\|\omega\|: 0 \neq \omega \in \Omega)$.

$$
\frac{\left|C_{T}(\mu)\right|}{|T|} \leq \frac{\|\mu\|}{(k q-1) \cdot l}
$$

Proof. For $k=1$ the proposition is exactly Lemma 5.1 in [8]. Suppose $k<1$. It is shown in $[8$, p.293] that

$$
\frac{\left|C_{T}(\mu)\right|}{|T|} \leq \frac{\|\mu\|}{\min _{0 \neq y \in \Omega}\|F(y)-y\|},
$$

so it suffices to set up a suitable lower bound for the denominator. As in $[8,5.1]$, we have here 


$$
|| F(y)-y\left\|\geq|(|| F(y)||-|| y||)| \geq\left(\frac{q}{t}-1\right) \cdot\right\| y \| \geq(k q-1) \cdot l
$$

by Lemma 6.1. (If $k=1$ then $\|F(y)\|=q \cdot\|y\|$ and $k=1 / t$ where $t$ is as in Lemma 6.1.)

Corollary 6.3. Let $k, T$ be as in Proposition 6.2. Suppose that $\mu \neq 0$.

(1) If $q>\frac{1}{k}+\frac{\|\mu\|}{k \cdot l}$ then $\left(s_{\mu}, 1_{T}\right)=0$.

(2) If $q>\frac{1}{k}+\frac{2\|\mu\|}{k l}$ then $T$ separates the weights of $W \mu$.

Proof. (1) Suppose the contrary. Then $C_{T}(w(\mu))=T$ for some $w \in W$. By Proposition 6.2 , we have $k q-1 \leq \frac{\|w(\mu)\|}{l}$. This is a contradiction as $\|w(\mu)\|=\|\mu\|$. (2) Suppose that $w(\mu) \neq w^{\prime}(\mu)$ for $w, w^{\prime} \in W$ and $\left.w(\mu)\right|_{T}$ and $\left.w^{\prime}(\mu)\right|_{T}$ are the same characters of $T$. Then $\left.\left(w(\mu)-w^{\prime}(\mu)\right)\right|_{T}=1$, that is, $C_{T}\left(w(\mu)-w^{\prime}(\mu)\right)=T$. As $\left\|w(\mu)-w^{\prime}(\mu)\right\| \leq 2\|\mu\|$, the result follows from (1).

Corollary 6.4. Let $M$ be an irreducible G-module. Then there are only finitely many groups $G=G(q)$ such that $\left.M\right|_{G} \otimes S t_{G}$ is not a sum of regular characters.

Remark. The values of $l$ for every simple algebraic group are known to be the following: $A_{n}: l=n /(n+1) ; B_{n}(n \geq 3): l=\min (1, n / 4) ; C_{n}(n \geq 2), D_{n}(n \geq 4): l=1$; $E_{6}: l=4 / 3 ; E_{7}: l=3 / 2 ; E_{8}: l=2 ; F_{4}: l=1 ; G_{2}: l=2 / 3$. We are indebted to Pham Huu Tiep for communicating us these.

Acknowledgment. The second author gratefully acknowledges a partial support from the Leverhulme Trust (grant EM/2006/0030).

\section{REFERENCES}

[1] J. Ballard, Projective modules for finite Chevalley groups, Trans. Amer. Math. Soc. 245(1978), $221-249$.

[2] N. Bourbaki, Groupes et algebres de Lie, Chaps. IV-VI, Hermann, Paris, 1968.

[3] R. Carter, Finite groups of Lie type: Conjugacy classes and complex characters, Wiley, 1985.

[4] C. W. Curtis, I. Reiner, Methods of representation theory with applications to finite groups and orders, Vol. 1,2, John Wiley and Sons, New York e.a., 1981.

[5] L. Chastkofsky, Projective characters for finite Chevalley groups, J. Algebra 69(1981), 347 - 357.

[6] P. Deligne and G. Lusztig, Duality for representations of a reductive group over a finite field, II, J. Algebra 81(1983), $540-545$.

[7] F. Digne and J. Michel, Representations of finite groups of Lie type, London Math. Soc. Student Texts 21, Cambridge University Press, 1991.

[8] R. Guralnick and Pham Huu Tiep, Finite simple unimodular groups, J. Group Theory 6(2003), $271-310$.

[9] G. Hiss and A. Zalesski, The Weil-Steinberg character of finite classical groups, J. Representation Theory (submitted)

[10] G. Lusztig, The discrete series of $\mathrm{GL}_{n}$ over a finite field, Annales of Mathematical Studies, Number 81, Princeton University Press, Princeton, New Jersey, 1974.

[11] J. Humphreys, Deligne-Lustzig characters and principal indecomposable modules, J. Algebra 62(1980), 299-303.

[12] J. Humphreys, Modular representations of finite groups of Lie type, Cambridge Univ. press, Cambridge, 2006.

[13] J. C. Jantzen, Über das Decompositionsverhalten gewisser modularer Darstellungen halbeinfacher Gruppen und ihrer Lie-algebren, J. Algebra 49(1977), 441 - 469. 
[14] G. Seitz, Bounds for dimensions of weight Spaces of Maximal Tori", In: 'Linear Algebraic Groups and Their Representations, Contemporary Mathematics Vol.153, American Mathematical Society, 1993', 157-161.

[15] T. Springer, Relèvement de Brauer et represéntations paraboliques de $G L_{n}\left(\mathbf{F}_{q}\right)$ (d'aprés G. Lusztig), Séminaire BOURBAKI, 26e annèe 1973/74, $n^{\circ} 441,89$ - 113.

[16] T. Springer and R. Steinberg, Conjugacy classes, In: A. Borel et al,'Seminar on algebraic groups and related finite groups', Springer-Verlag, Berlin, 1970.

[17] R. Steinberg, Lectures on Chevalley groups, mimeographed lecture notes, Yale Univ. Math. Dept., New Haven, Conn., 1968.

[18] W.J. Wong, Representations of Chevalley groups in characteristic p, Nagoya J. Math. 45(1971), 39-78.

G.H.: Lehrstuhl D FÜr Mathematik, RWTH Aachen University, 52056 Aachen, Germany

A.Z.: Departimento di Matematica e Applicazioni, Universitóegli Studi di Milano-Bicocca, Milano, via Roberto Cozzi 53, 20125, Italy

E-mail address: G.H.: gerhard.hiss@math.rwth-aachen.de

E-mail address: A.Z.: alexandre.zalesski@gmail.com 